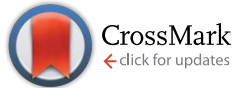

Cite this: Chem. Sci., 2016, 7, 4264

\section{"Rate-limited effect" of reverse intersystem crossing process: the key for tuning thermally activated delayed fluorescence lifetime and efficiency roll-off of organic light emitting diodes $\uparrow$}

\author{
Xinyi Cai, Xianglong Li, Gaozhan Xie, Zuozheng He, Kuo Gao, Kunkun Liu, \\ Dongcheng Chen, Yong Cao and Shi-Jian Su*
}

\begin{abstract}
Issues concerning excited state lifetime $\left(\tau_{\text {TADF }}\right)$ tuning of thermally activated delayed fluorescence (TADF) materials are critical for organic light emitting diode (OLED) applications and other specific fields. For TADF-OLEDs, employing emitters with a short $\tau_{\text {TADF }}$ gives rise to suppressed singlet-triplet annihilation (STA) and triplet-triplet annihilation (TTA), leading to reduced efficiency roll-off at practical relevant brightness (100 and $1000 \mathrm{~cd} \mathrm{~m}^{-2}$ for display and illumination applications, respectively). Through molecular design, exciton dynamic process rate constants including fluorescence $\left(k_{\mathrm{F}}\right)$, intersystem crossing $\left(k_{\mathrm{ISC}}\right)$, internal conversion $\left(k_{\mathrm{IC}}\right)$ and reverse intersystem crossing $\left(k_{\mathrm{RISC}}\right)$ are selectively altered, affording four representative TADF emitters. Based on lifetime and quantum yield measurements, $k_{\mathrm{F}}, k_{\mathrm{ISC}}$, $k_{\mathrm{IC}}$ and $k_{\mathrm{RISC}}$ are calculated for four emitters and their interrelationship matches corrected timedependent density functional theory simulation. Among them, even with a small $k_{\mathrm{F}}$, low photoluminescence quantum efficiency $(\Phi)$ and large $k_{\mathrm{ISC}}$, molecules with a small singlet-triplet splitting energy $\left(\Delta E_{\mathrm{ST}}\right)$ and lowest charge transfer triplet excited state $\left({ }^{3} \mathrm{CT}\right)$ eventuate in shortening the $\tau_{\mathrm{TADF}}$. Herein, $k_{\mathrm{RISC}}$, which is inversely proportional to $\Delta E_{\mathrm{ST}}$, turns out to be the rate-limited factor in tuning the $\tau_{\text {TADF }}$ ("rate limited effect" of the RISC process). As revealed by flexible potential surface scanning, PyCNACR exhibited a moderate $k_{\mathrm{F}}$, reduced $k_{\mathrm{IC}}$ and enlarged $k_{\mathrm{RISC}}$, resulting in a short $\tau_{\text {TADF }}$ and a moderate $\Phi$ with orange-red emission. OLEDs containing PyCN-ACR as the emitting guest achieved orange-red TADF-OLEDs with an emission peak at $590 \mathrm{~nm}$ and the best external quantum efficiencies (EQEs) of $12.4 \% / 9.9 \% / 5.1 \%$ at practical luminances of $100 / 1000 / 10000 \mathrm{~cd} \mathrm{~m}^{-2}$.
\end{abstract}

Received 3rd February 2016 Accepted 4th March 2016

DOI: $10.1039 / c 6 s c 00542 j$

www.rsc.org/chemicalscience relevant) and $1000 \mathrm{~cd} \mathrm{~m}^{-2}$ (illumination relevant) is of great importance. ${ }^{5}$ An alternative approach for $100 \%$ IQE in OLEDs is utilizing thermally activated delayed fluorescence (TADF) materials. ${ }^{6} 75 \%$ of the electric-generated triplet excitons can be up-converted to the radiative singlet state to yield delayed fluorescence thanks to a very small $\Delta E_{\mathrm{ST}}{ }^{7-9}$ Analogous to $\mathrm{PH}$ OLEDs, however, the EQEs of TADF-OLEDs at high brightness are also subjected to reduction by a large margin. ${ }^{\mathbf{1 0 , 1 1}}$ Except for TTA, a higher singlet exciton density than Ir-based PH-OLEDs from an efficient RISC process produces a non-negligible STA. ${ }^{\mathbf{1 2 , 1 3}}$ These superimposed factors make the reduced efficiency roll-off of TADF-OLEDs challenging on the march towards future high brightness applications (Scheme 1).

The short excited state lifetime of TADF materials $\left(\tau_{\mathrm{TADF}}\right)$ is important to reduce the efficiency roll-off of TADF-OLEDs by suppression of the TTA and STA. ${ }^{4}$ For TADF emitters, the rate constants of fluorescence $\left(k_{\mathrm{F}}\right)$, intersystem crossing $\left(k_{\mathrm{ISC}}\right)$, reverse intersystem crossing ( $\left.k_{\mathrm{RISC}}\right)$, internal conversion $\left(k_{\mathrm{IC}}\right)$, and non-radiative decay from the triplet state $\left(k_{\mathrm{nr}}^{\mathrm{T}}\right)$ play roles in exciton dynamic processes and could govern $\tau_{\text {TADF }}$. Given that 


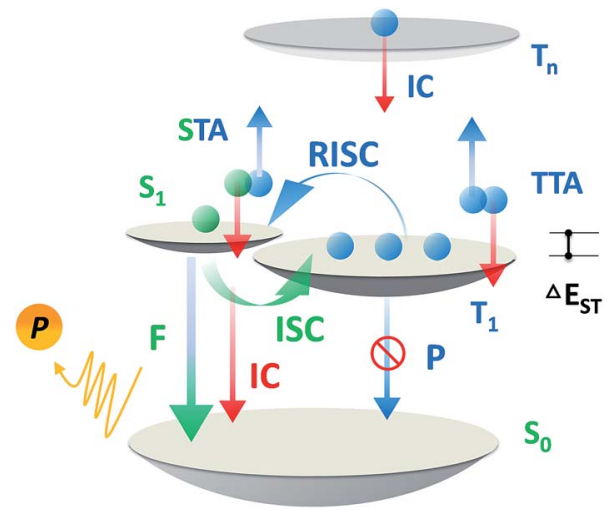

Scheme 1 Exciton dynamic processes for a typical TADF emitter in OLEDs. Singlet and triplet excitons are generated in a $1: 3$ ratio depending on the spin degeneracy. F, P, ISC, IC, STA, TTA and RISC are abbreviations of fluorescence, phosphorescence, intersystem crossing, internal conversion, singlet-triplet annihilation, triplet-triplet annihilation, and reverse intersystem crossing.

some of these factors are also involved in the EQE annihilation model of TADF-OLEDs (2c, ESI $\dagger$ ), ${ }^{13}$ it is reasonable to deduce that tuning these factors via chemical modification could eventuate in various EQE roll-off curves even in exactly the same device architecture. Notably, it can be complicated to comprehend and design materials taking all factors into account, and the critical point for tuning $\tau_{\mathrm{TADF}}$ remains unclarified. Therefore, selectively tuning these factors does help to find out the key to govern $\tau_{\mathrm{TADF}}$, and ultimately not only assists in promoting efficiencies of TADF-OLEDs at practically relevant brightness, but can be used for reference towards material design in other specific fields where excited lifetime tuning is required.

To handle this, the governing of these factors should be linked to molecular design. To our knowledge, $k_{\mathrm{RISC}}$ is proportional to $\exp \left(-\Delta E_{\mathrm{ST}} / k_{\mathrm{B}} T\right),{ }^{8}$ where $k_{\mathrm{B}}$ denotes the Boltzmann constant and $T$ denotes temperature. $\Delta E_{\mathrm{ST}}$ is proportional to the orbital overlap $\left(\mathrm{S}_{\mathrm{if}}\right)$ of the initial $\left(\Phi_{\mathrm{i}}\right)$ and final $\left(\Phi_{\mathrm{f}}\right)$ state wavefunctions of the $\mathrm{S}_{1}$ transition. Meanwhile, $k_{\mathrm{F}}$ is related to the transition integral $\left(\mathrm{H}_{\mathrm{if}}\right)$ and is roughly proportional to $\mathrm{S}_{\mathrm{if}}{ }^{\mathbf{1 4 1 5}}$ Therefore, $k_{\mathrm{F}}$ and $k_{\mathrm{RISC}}$ cannot be cut apart for TADF emitters, because a large $k_{\mathrm{F}}$ originating from a large frontier orbital overlap could enlarge $\Delta E_{\mathrm{ST}}$ and reduce $k_{\mathrm{RISC}}$. A convenient approach to tune $k_{\mathrm{F}}$ and $k_{\mathrm{RISC}}$ is introducing twisted intramolecular charge transfer (TICT) systems with different dihedral angles between electron donating and withdrawing moieties. ${ }^{7,9}$ Enlarging the dihedral angles between D-A systems minimizes the transition integral, resulting in reduced $k_{\mathrm{F}}$ and $\Delta E_{\mathrm{ST}}$ and vice versa. For emitters without heavy atoms, metals or halogens, $k_{\mathrm{ISC}}$ can be tuned using emitters with $\pi-\pi^{*}$ or $\mathrm{n}-\pi^{*}$ transition character, for the latter ones facilitate the forbidden transition from the singlet to triplet excited state that violates spin conservation to enhance $k_{\mathrm{ISC}}{ }^{16-18}$ For $k_{\mathrm{IC}}$, utilizing rigid chemical moieties and compact molecular structure design can remarkably suppress non-radiative channels. Notably, nonradiative processes in the triplet state are much slower than others given that $T_{1}$ is relatively stable for pure aromatic compounds, and the influence of $k_{\mathrm{nr}}^{\mathrm{T}}$ can be temporarily ignored for simplicity ${ }^{19}$ By the aforementioned criteria, molecules exhibiting specific characters can be designed.

In this work, instead of starting from host engineering or device architecture management, we design four typical TADF emitters to elaborate the connection among $\tau_{\mathrm{TADF}}$ governing, device efficiency roll-off control and molecular design. Among the diverse chemical moieties, carbonyl-type compounds are chosen for their special $\mathrm{n}-\pi^{*}$ transition features, which inevitably renders a small $k_{\mathrm{F}}$ and a significant ISC process..$^{17,20-22}$ Along this line of thinking, the benzil (DC) moiety is selected. On one hand, its orthogonal $\mathrm{n}$ and $\pi^{*}$ orbitals diminishes the exchange integral of the highest occupied molecular orbital (HOMO) and the lowest unoccupied molecular orbital (LUMO), enabling a fairly small $\Delta E_{\mathrm{ST}}$ around $0.1-0.2 \mathrm{eV} .{ }^{14,23}$ Additionally, alternation of the electronic configuration can be realized via simple modification of the DC core, affording another representative radiative conducive $\pi-\pi *$-favoured constituent. The replacement of benzil by dicyano-pyrazine (PyCN) offers typical fluorophores with a large $k_{\mathrm{F}}$, reduced $k_{\mathrm{IC}}$ and $k_{\mathrm{ISC}} \cdot{ }^{24,25}$ Aside from the acceptor cores, the donors are fixed on TC (3,6-di-tertbutylcarbazole) and ACR (9,10-dihydroacridine) moieties, respectively. The six-membered ACR ring induces enlarged D-A twisting compared with the five-membered TC heterocycle, ${ }^{9}$ leading to a reduced $\Delta E_{\mathrm{ST}}$ and enhanced molecular rigidity for DC-ACR and PyCN-ACR. The aforementioned design thinking embodies four distinct and representative types of molecules as part of a large class of TADF emitters with analogous characters. The influence of molecular design on governing $\tau_{\text {TADF }}$ by tuning $k_{\mathrm{F}}, k_{\mathrm{ISC}}, k_{\mathrm{IC}}$ and $k_{\mathrm{RISC}}$ are investigated thoroughly by examining the photophysical properties and quantum chemical simulations. Lastly, OLEDs are fabricated to ascertain the affiliation between molecular design and device performance concerning efficiency roll-off (Fig. 1).

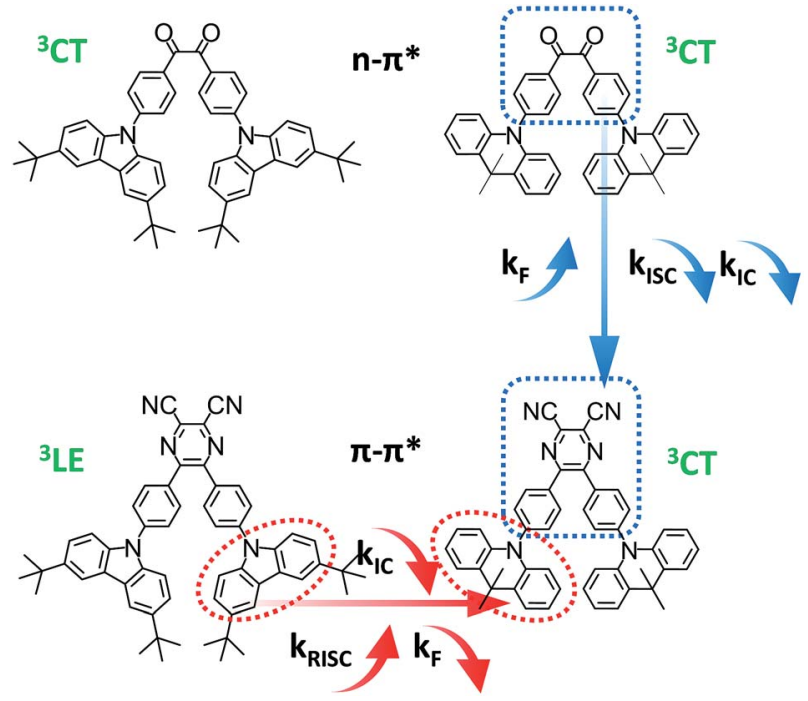

Fig. 1 Molecular structures of the investigated compounds and molecular design for tuning $k_{\mathrm{F}}, k_{\mathrm{IC}}, k_{\mathrm{ISC}}$ and $k_{\mathrm{RISC}}$. 


\section{Results and discussion}

\section{Computation simulation}

In order to demonstrate the influence of molecular design on governing $\tau_{\mathrm{TADF}}$ by tuning $k_{\mathrm{F}}, k_{\mathrm{ISC}}, k_{\mathrm{IC}}$ and $k_{\mathrm{RISC}}$, comprehensive pictures of the molecules should be provided. Through adopting the appropriate methodology, density functional theory (DFT) and time-dependent density functional theory (TD-DFT) simulations can provide relatively reasonable descriptions of the investigated molecules. ${ }^{\mathbf{2 6 - 2 8}}$ Initially, the ground state $\left(\mathrm{S}_{0}\right)$ geometries of the four emitters were optimized at the B3LYP/6$31 \mathrm{G}^{*}$ level. ${ }^{28,29}$ Different from the $\mathrm{S}_{0}$ geometry, $\mathrm{S}_{1}$ geometries for ICT molecules strongly depend on the exchange-exchange (XC) functional. ${ }^{30}$ Besides, selection of a proper functional to depict the vertical absorption transition energy $\left(E_{\mathrm{VA}}\right)$ and emission energy $\left(E_{\mathrm{VE}}\right)$ is required, because non-hybrid functionals neglect long-range coulombic attraction and underrate the CT transition energy while pure Hartree-Fock (HF) functionals suffer from electron correlation problems and overrate the excited energy. ${ }^{31}$ Lastly, the configuration interaction (CI) description of the $\mathrm{S}_{1}$ transition on coupling between the charge transfer excited state (CT) or locally excited state (LE) also relies on the XC functional. ${ }^{9}$ To simplify the simulation, a semi-empirical approach using experimental $E_{\mathrm{VA}}\left(\mathrm{S}_{1}\right)$ to find the optimal percentage of the $\mathrm{HF}$ fraction (OHF) into functionals was adopted. Different HF\% functionals were employed to compute $E_{\mathrm{VA}}\left(\mathrm{S}_{1}\right)$ based on the optimized $\mathrm{S}_{0}$ geometry. $\mathrm{HF} \%$ was plotted against $E_{\mathrm{VA}}\left(\mathrm{S}_{1}\right)$ and the relationship was established (Fig. $\mathrm{S} 7 \dagger$ ). The optimized HF\% (OHF) was determined to be close to $42 \%$ (BMK) by fitting the simulated $E_{\mathrm{VA}}\left(\mathrm{S}_{1}\right)$ with well-defined CT absorption peaks from the ultraviolet-visible (UV-vis) spectroscopy measurement of all molecules. ${ }^{32}$ As $S_{1}$ is sensitive towards $\mathrm{XC}$ functionals and solvent effects, it is necessary to optimize the $S_{1}$ geometries, calculate $E_{\mathrm{VE}}$ and analyze the configuration interaction (CI) description at the $\mathrm{BMK} / 6-31 \mathrm{G}^{*}$ level utilizing the polarized continuum model (PCM) and so potential deviation can be minimized..$^{3,34}$

The simulated results of the four $\mathrm{D}-\pi-\mathrm{A}-\pi-\mathrm{D}$ compounds can be divided into two categories (Scheme 2). For the $S_{0}$ and $S_{1}$ geometries, detailed parameters including the bond length $(l)$ and dihedral angle $(\alpha$ or $\beta$ ) are listed in Table 1 . To visualize the frontier orbital transition of all compounds, natural transition orbital (NTO) analyses in view of singular value decomposition of the one-particle transition density matrix were carried out to

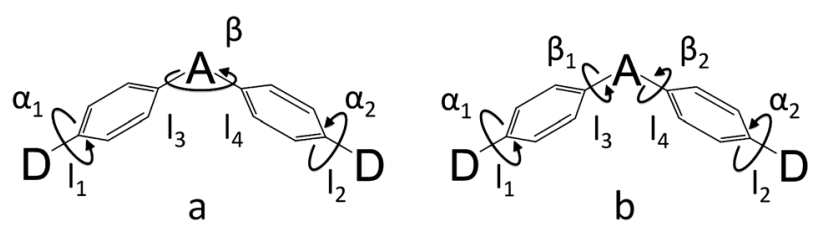

Scheme 2 Two models for donor- $\pi$-acceptor $-\pi$-donor (D- $\pi-A-$ $\pi-D)$ molecules ( $(a)$ for DC-TC and DC-ACR, (b) for PyCN-TC and PyCN-ACR). $\alpha$ and $\beta$ denote dihedral angles. $l_{1}$ and $l_{2}$ denote the bond lengths between the donor and phenyl bridge, while $l_{3}$ and $l_{4}$ denote the bond lengths between the acceptor and phenyl bridge. comprehend the electron transitions, as NTO analyses provide fewer orbital pairs with maximum eigenvalues and simplify our interpretation. ${ }^{35,36}$ The geometries and NTOs of the four molecules are presented (Fig. 2 and 3), respectively.

In the $\mathrm{S}_{0}$ geometry, the six-membered ACR offers a large steric hindrance effect from repulsion between the adjacent hydrogen atoms and $\alpha=89^{\circ}$ was estimated, much larger than that between $\mathrm{TC}$ and $\mathrm{Ph}$ (around $47^{\circ}$ ). The benzil moiety twists and avoids forming a flat surface in $\mathrm{S}_{0}$. $\beta$ between PyCN and the $\mathrm{Ph}$ bridge lies around $34^{\circ}$ (Fig. $\mathrm{S} 6 \dagger$ ) and twists to avoid repulsion with the ortho-substituted $\mathrm{Ph}$ ring. For vertical electron excitation, the $S_{0}$ to $S_{1}$ transition of DC-TC locates at the benzil moiety and has a much smaller oscillator strength $(f \approx 0)$ than $\mathrm{S}_{2}$; the latter is regarded as $\mathrm{S}_{1}$ in the following discussion. The lowest unoccupied natural transition orbitals (LUNTOs) of DCTC and PyCN-TC predominantly localize on the DC and PyCN cores and slightly delocalize over the adjacent $\mathrm{Ph}$ ring. The highest occupied natural transition orbitals (HONTOs) distribute mainly on both TC-Ph arms and a small amount on the acceptor plane. Owing to the enlarged $\alpha$, DC-ACR and PyCN-ACR have small orbital overlap, with the HONTO localizing mostly on ACR and the LUNTO localizing on the DC and PyCN-2Ph centers. The decreased coplanarity between the donor and acceptor prohibits nitrogen lone pair electrons from delocalizing over the whole molecule. In view of the more limited HONTO and LUNTO overlap for DC-ACR and PyCNACR, a much smaller $f$ can be anticipated than DC-TC and PyCN-TC.

In $S_{1}$, the bond lengths $\left(l_{1}, l_{2}, l_{3}, l_{4}\right)$ show rather small variations in comparison with the $S_{0}$ geometry. However, $\alpha$ and $\beta$ change significantly, suggesting molecular geometry relaxation happens in $\mathrm{S}_{1}$. $\beta$ decreases from $44.8^{\circ}$ to $3.0^{\circ}$ for DC-TC and from $49.5^{\circ}$ to $5.8^{\circ}$ for DC-ACR. A similar trend is also observed for PyCN-TC, as both $\beta_{1}$ and $\beta_{2}$ show a slight decrease to induce a more conjugated acceptor plane. However, for PyCN-ACR, $\beta_{2}$ decreases from $35.1^{\circ}$ to $15.8^{\circ}$ while $\beta_{1}$ increases from $35.1^{\circ}$ to $40.7^{\circ}$, which can be explained by a more compact geometry compared with PyCN-TC. To twist a rigid ACR-Ph arm inevitably enhances the steric hindrance of the other, which is forced to avert close interaction between the adjacent ACR-Ph arms. The images comparing the $S_{0}$ and $S_{1}$ geometries (Fig. 2) clearly illustrate a degree of deformation and qualitatively depict more enhanced rigidity with smaller discrepancy. The coplanarization of acceptors and the internal twist of donors can be interpreted by the minimum overlap rule, which drives more complete charge separation of the donor and acceptor so as to lower the CT state energy. ${ }^{37}$ The flexible benzil acceptor tends to elongate conjugation, and this and the slightly increased $\alpha$ both contribute to separate further the HONTO and LUNTO and weakens electronic coupling between the donor and acceptor. ${ }^{38-40}$ Compared with DC-TC and DC-ACR, more suppressed non-radiative deactivation processes can be expected for PyCN-TC and PyCN-ACR showing lower geometry deformation in $\mathrm{S}_{1}$.

In the optimal $S_{1}$ geometries, NTO transitions analogous to those in $\mathrm{S}_{0}$ are observed for DC-TC and PyCN-TC but brokensymmetry effects are noted for DC-ACR and PyCN-ACR. 
Table 1 Calculated twisting angles $\left(\alpha_{1}, \alpha_{2}\right.$ and $\left.\beta_{1}, \beta_{2}\right)$, bond lengths $\left(l_{1}, l_{2}\right.$, and $\left.l_{3}, l_{4}\right)$, transition energies $\left(E_{\mathrm{VA}}\right.$ and $E_{\mathrm{VE}}$ based on OHF functionals), and oscillator strengths ( $f_{\mathrm{VA}}$ and $f_{\mathrm{VE}}$ based on OHF functionals) of all investigated molecules in the gas phase in $\mathrm{S}_{0}$-optimized geometries and in solvents in $\mathrm{S}_{1}$-optimized geometries

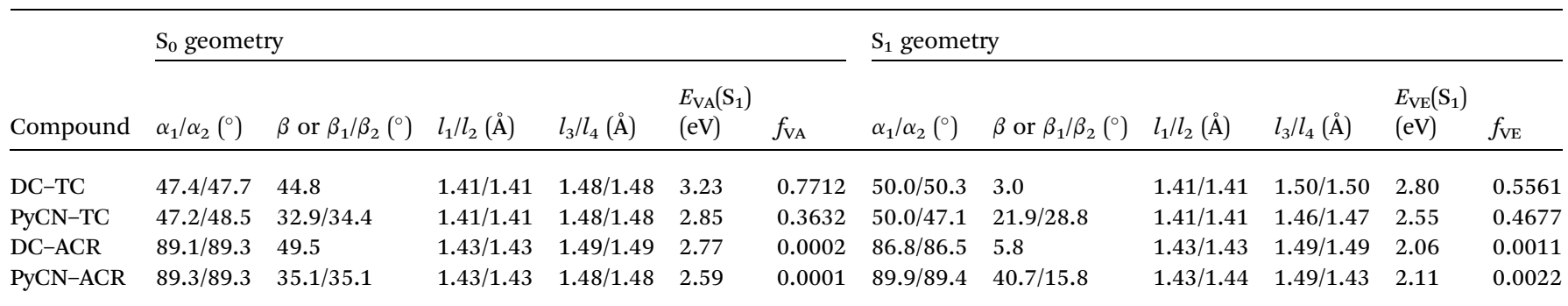

Followed by geometry relaxation, the degrees of HONTO and LUNTO overlap for DC-TC and PyCN-TC still far exceed those of DC-ACR and PyCN-ACR, indicating a larger $k_{\mathrm{F}}$ for the former ones. It is noteworthy that despite partially overlapping centres on the benzil moiety for the HONTO and LUNTO of DC-ACR, the $\mathrm{n}-\pi^{*}$ transition nature of the benzil moiety hardly contributes to the overall $f$ value. ${ }^{21,22}$ A $f$ value roughly equal to zero is observed for such a typical $n-\pi *$ transition in both the $S_{0}$ and $\mathrm{S}_{1}$ geometries of the DC center, explaining the negligible $f_{\mathrm{VA}}$ and $f_{\mathrm{VE}}$ for DC-ACR (Fig. S5 and Table S1 $\dagger$ ).

To interpret the energy level coupling relationship, calculation of zero-zero energy levels $\left(E_{0-0}\right)$ is critical. A systematic comparison of $E_{\mathrm{VA}}\left(\mathrm{S}_{1}\right), E_{\mathrm{VE}}\left(\mathrm{S}_{1}\right), E_{0-0}\left({ }^{1} \mathrm{CT}\right), E_{0-0}\left({ }^{3} \mathrm{CT}\right), E_{0-0}\left({ }^{3} \mathrm{LE}\right)$ and $\Delta E_{\mathrm{ST}}$ is listed (Table $\mathrm{S} 2 \dagger$ ). According to the Franck-Condon principle, the average values of $E_{\mathrm{VA}}\left(\mathrm{S}_{1}\right)$ and $E_{\mathrm{VE}}\left(\mathrm{S}_{1}\right)$ approximate the $E_{0-0}$ energy level by eqn (1) and reproduce the experimental data with small deviation (Fig. S7†). ${ }^{41}$

$$
E_{0-0}\left({ }^{1} \mathrm{CT}\right)=\left(E_{\mathrm{VA}}\left(\mathrm{S}_{1}\right)+E_{\mathrm{VE}}\left(\mathrm{S}_{1}\right)\right) / 2
$$

For analyzing the RISC process, coupling between the CT and LE triplet states should be carefully defined. The lower-lying
${ }^{3} \mathrm{LE}$ state than the ${ }^{3} \mathrm{CT}$ state ought to decrease the rate constants of the RISC process because of the slow reverse internal conversion (RIC) rate from the ${ }^{3} \mathrm{LE}$ state to the ${ }^{3} \mathrm{CT}$ state. ${ }^{9}$ Both $E_{0-0}\left({ }^{3} \mathrm{CT}\right)$ and $E_{0-0}\left({ }^{3} \mathrm{LE}\right)$ were computed independently based on eqn (2) and (3) (Scheme 3). ${ }^{42}$

$$
\begin{gathered}
E_{0-0}\left({ }^{3} \mathrm{CT}\right)=E_{0-0}\left(\mathrm{~S}_{1}\right)-\left(E_{\mathrm{VA}}\left(\mathrm{S}_{1}, \mathrm{OHF}\right)\right. \\
\left.\quad-E_{\mathrm{VA}}\left(\mathrm{S}_{1}, \mathrm{OHF}\right) / E_{\mathrm{VA}}\left(\mathrm{S}_{1}, \mathrm{BLYP}\right) \times E_{\mathrm{VA}}\left(\mathrm{T}_{1}, \mathrm{BLYP}\right)\right) \\
E_{0-0}\left({ }^{3} \mathrm{LE}\right)=E_{\mathrm{VA}}\left(\mathrm{T}_{1}\right) /\left(E _ { \mathrm { VA } } \left(\mathrm{S}_{1}\right.\right. \\
\left.\mathrm{OHF}) / E_{\mathrm{VA}}\left(\mathrm{S}_{1}, \mathrm{BLYP}\right)\right)-0.09
\end{gathered}
$$

Base on the calculated $E_{0-0}\left({ }^{3} \mathrm{CT}\right)$ and $E_{0-0}\left({ }^{3} \mathrm{LE}\right)$, the lower energy level turns out to be the lowest $\mathrm{T}_{1}$ state and $\Delta E_{\mathrm{ST}}$ can be obtained. The reverse internal conversion (IC) rate is determined by the energy gap and the thermal equilibrium process between ${ }^{3} \mathrm{LE}$ and the higher ${ }^{3} \mathrm{CT} .{ }^{9} \mathrm{~A}$ lower-lying ${ }^{3} \mathrm{LE}$ state than the ${ }^{3} \mathrm{CT}$ state impedes an efficient RISC process and enlarges $\Delta E_{\mathrm{ST}}{ }^{43}$ Triplet exciton generation at the ${ }^{3} \mathrm{LE}$ state is hardly upconverted into the radiative ${ }^{1} \mathrm{CT}$ state, which leads to a high triplet exciton density. Except for PyCN-TC, all the evaluated

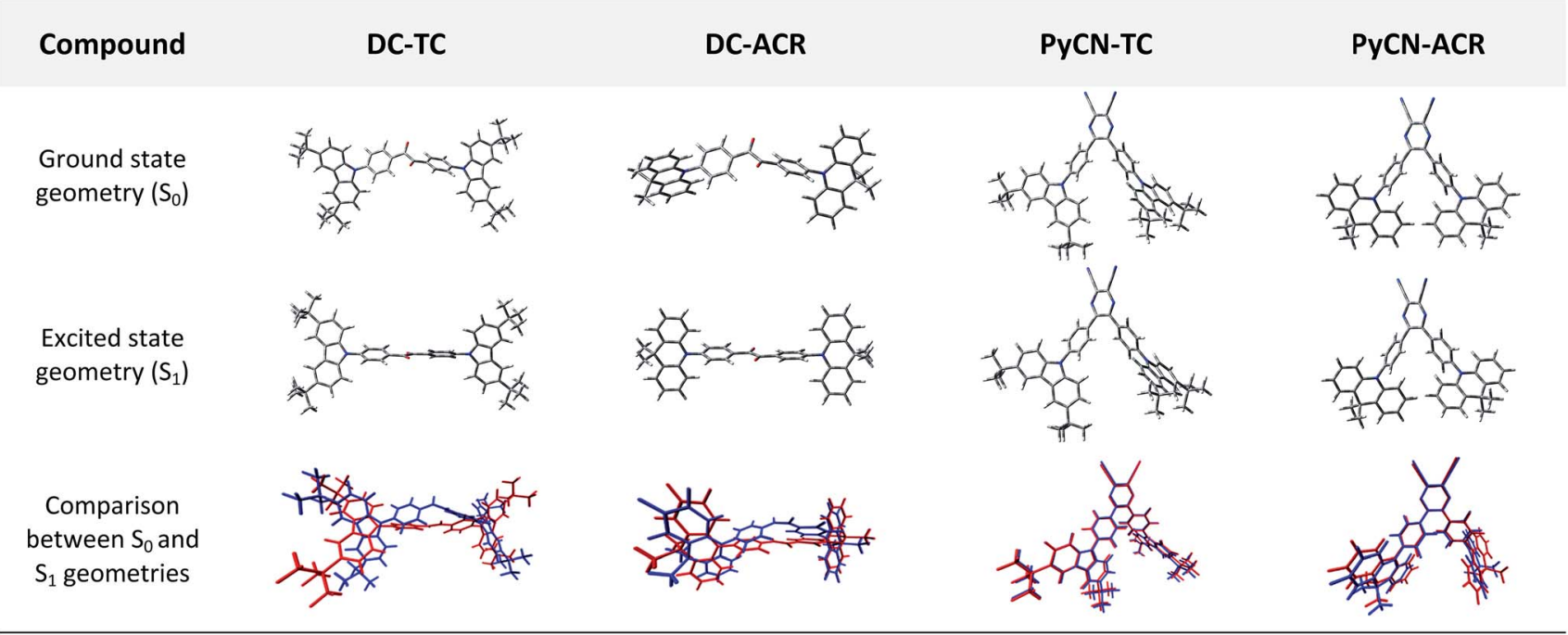

Fig. 2 Front views of the DFT-optimized ground state geometries from B3LYP/6-31G* in the gas phase and excited state geometries from BMK/ 6-31G* in toluene using the PCM model for the four compounds. The bottom geometries are intuitive pictures comparing the $\mathrm{S}_{0}$ (blue) and $\mathrm{S}_{1}$ (red) geometries with minimum root-mean-square-deviation (RMSD). 


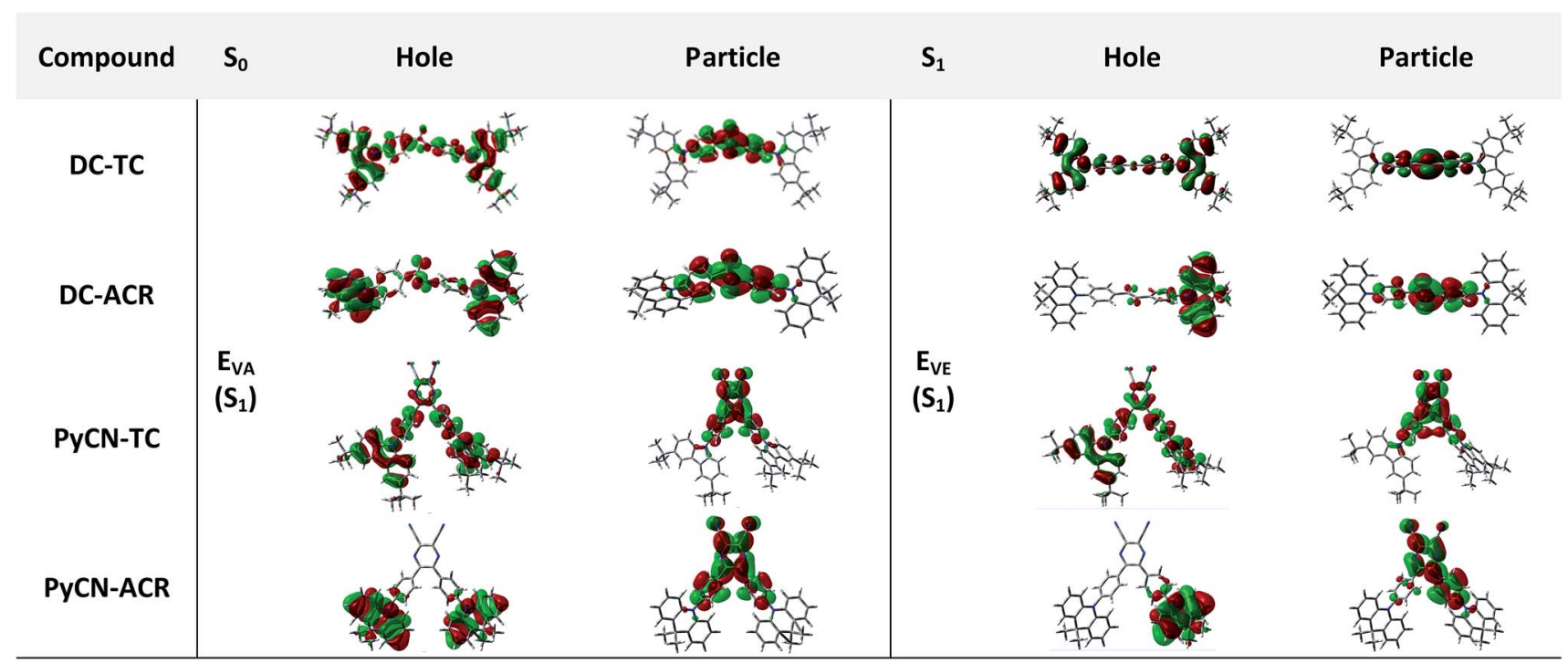

Fig. 3 Natural transition orbital (NTO) analyses of the four investigated molecules. Hole and particle distributions of the HONTO and LUNTO of the $S_{0}$ and $S_{1}$ geometries are presented for intuitive comparison.

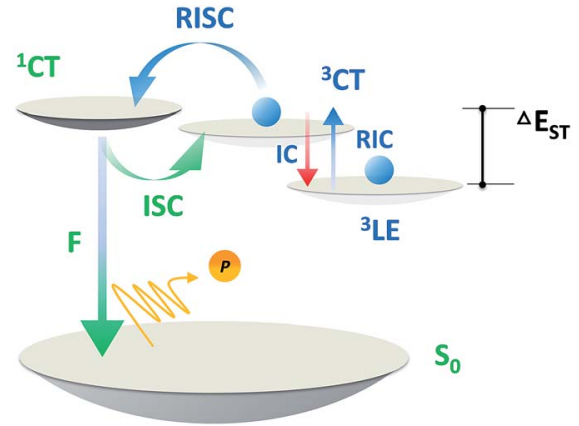

Scheme 3 Exciton dynamic process for TADF emitters with and without a local triplet excited state $\left({ }^{3} \mathrm{LE}\right)$. RIC denotes reverse internal conversion.

$E_{0-0}\left({ }^{3} \mathrm{LE}\right)$ are higher than $E_{0-0}\left({ }^{3} \mathrm{CT}\right)$. The $E_{0-0}\left({ }^{3} \mathrm{LE}\right)$ of PyCN-TC is lower than $E_{0-0}\left({ }^{3} \mathrm{CT}\right)$ by $0.31 \mathrm{eV}$ and could be the predominant energy level forming triplet excitons under optical excitation or in EL devices. The difference between $E_{0-0}\left({ }^{1} \mathrm{CT}\right)$ and lowest $\mathrm{T}_{1}$ defines $\Delta E_{\mathrm{ST}}$, which is proportional to the degree of orbital overlap $\left(S_{\text {if }}\right)$. With qualitative analyses of the overlap extent of HONTO and LUNTO, larger $\Delta E_{\mathrm{ST}}$ values are calculated for DCTC (0.21) and PyCN-TC (0.46) than DC-ACR (0.01) and PyCNACR (0.01) because the almost orthogonal dihedral angles between ACR and the acceptor cores induce a more separated HONTO and LUNTO. Due to the existence of the low-lying ${ }^{3} \mathrm{LE}$ state, the calculated $\Delta E_{\mathrm{ST}}$ of PyCN-TC reaches $0.46 \mathrm{eV}$, which is over twice as much as its chemical precursor DC-TC. Since similar $\alpha$ values were observed for DC-TC and PyCN-TC, the distinction for $\Delta E_{\mathrm{ST}}$ can be explained by the different electronic configuration of the acceptor moiety. When the $n-\pi *$ transition DC center is chemically modified into PyCN, the increased $\pi-\pi$ * transition nature enhances the HONTO and LUNTO overlap, promoting a radiative transition process with a higher $k_{\mathrm{F}}$ and enlarged $\Delta E_{\mathrm{ST}}$. As mentioned, additional attention should be stressed on the CT and LE coupling of the triplet state in molecular design because ${ }^{3} \mathrm{LE}$ could intrinsically disable an efficient RISC process. The lowest ${ }^{3} \mathrm{CT}$ and small ${ }^{1} \mathrm{CT} /{ }^{3} \mathrm{CT}$ energy splitting enables an unhindered RISC process, which leads to reduction of the triplet exciton population under optical or electrical excitation.

\section{Photo-physical properties}

The investigated compounds DC-TC and DC-ACR were synthesized from commercially available starting materials in a one-step convenient Buchwald-Hartwig $\mathrm{C}-\mathrm{N}$ coupling reaction (Scheme 4). Based on DC-TC and DC-ACR, dicyano-pyrazine ring-closing products were easily produced followed by reactions with diaminomaleonitrile (DAMN) in acetic acid in high yields (>85\%), making these compounds cost effective. ${ }^{24,25}$ Their photophysical properties were studied in diluted solutions and doped films. Broad ICT absorption and photoluminescence (PL) bands were observed. For DC-TC and PyCN$\mathrm{TC}$, the vertical absorption transition exhibits a larger molar extinction coefficient $\left(\varepsilon=18.0-21.0 \mathrm{mM}^{-1} \mathrm{~cm}^{-1}\right)$ than those of DC-ACR and PyCN-ACR $\left(\varepsilon=2.5-5.0 \mathrm{mM}^{-1} \mathrm{~cm}^{-1}\right)$. The large $\varepsilon$ of DC-TC and PyCN-TC absorption can be understood by enhancement of the transition integral $\left(\mathrm{H}_{\mathrm{if}}\right)$ from large frontier orbital overlap. The solvatochromism effect on the absorption of all compounds was investigated (Fig. S10†). A fluctuating. molar extinction coefficient intensity as well as drifting of the absorption peak position was noted. Except for a solvation effect on the Franck-Condon transition, ${ }^{44}$ diverse $S_{0}$ molecular configuration distribution in various solvents can play a part. According to the DFT simulation geometry and the TD-DFT computed oscillator strength $(f)$, the orthogonally connected ACR and Ph inevitably give rise to a forbidden HONTO to LUNTO transition $(f \approx 0)$. Nevertheless, a vertical transition 


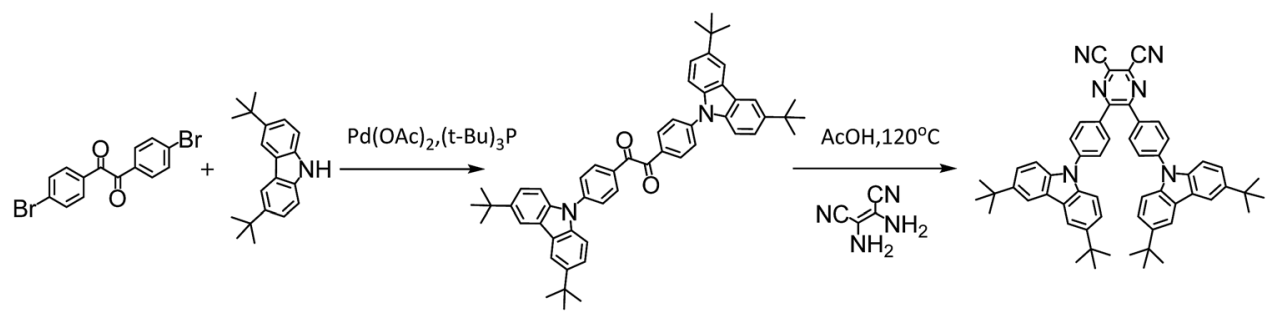

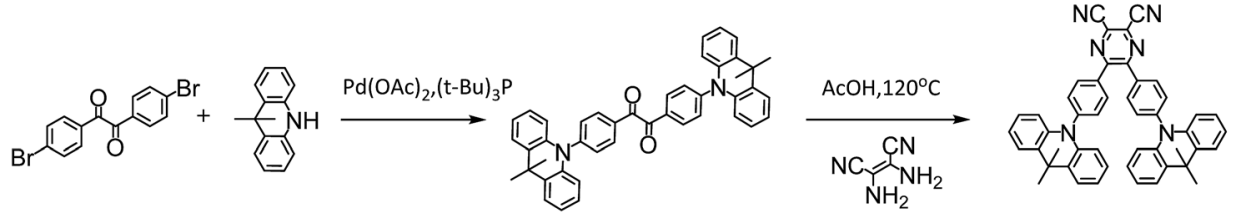

Scheme 4 Synthesis routes for all compounds and reaction conditions.

absorption with moderate $\varepsilon=2.5-5.0 \mathrm{mM}^{-1} \mathrm{~cm}^{-1}$ still can be detected, not to mention the moderate photoluminescence quantum yield (PLQY) of PyCN-ACR measured in the doped film state considering $k_{\mathrm{F}}$ is closely related to the $f$ value of ICT vertical absorption (Fig. 4). ${ }^{14}$

For greater understanding, flexible potential surface scans (full geometry optimization for each molecular conformation) were conducted for all molecules (Fig. 5). Due to symmetric double donor arms in the optimized $\mathrm{S}_{0}$ geometry, only one arm was selectively rotated for simplicity to explicate energy variation and molecular geometry distribution. Starting from the initial geometries, molecules were optimized (with $\Delta \alpha_{1}= \pm 5^{\circ}$ a step) to relax the irrational intramolecular interaction force, affording a more accurate vertical excitation energy and rotational energy barrier. Local energy maxima of DC-TC and PyCN-TC were obtained following a rotation $\Delta \alpha_{1}= \pm 40^{\circ}$. The energy discrepancy between the minimized and local maximum energy turns out to be $89 \mathrm{meV}$ for DC-TC and $75 \mathrm{meV}$ for PyCNTC. When undergoing the same twisting angle for DC-ACR and PyCN-ACR, the energy discrepancy enlarged to around $120 \mathrm{meV}$ for both molecules. The more rigid ACR-Ph arm than TC-Ph

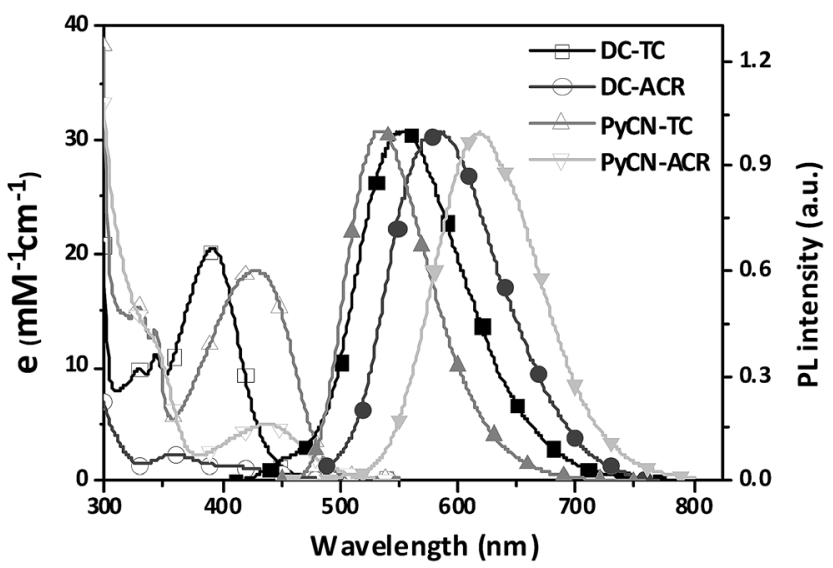

Fig. 4 UV-vis absorption and PL spectra of the investigated molecules measured in toluene (DC-ACR in $n$-hexane) $(0.05 \mathrm{mM})$ in air at room temperature. accounts for this, and a steeper potential energy surface can be expected. Due to the large dihedral angle at the minimized energy geometry, the ACR-Ph arm is relatively stationary, which leads to the almost forbidden transition. However, ambient temperature $(300 \mathrm{~K})$ with $25 \mathrm{meV}$ energy endows possible molecular rotation around $\Delta \alpha_{1}= \pm 25^{\circ} .{ }^{45,46}$ The transition is more permissible because the more planar $\mathrm{D}-\mathrm{A}$ conformation contributing to larger orbital overlap is found around the equilibrium conformation after activation by thermal energy. Theoretical absorption spectra of DC-ACR and PyCN-ACR were simulated based on $\mathrm{OHF} \%$ functionals according to different twisting angles $\left(\Delta \alpha_{1}\right)$ using Multiwfn (Fig. S11 $\dagger$ ). ${ }^{47}$ When the twisted angle $\left(\Delta \alpha_{1}\right)$ increased from $5-25^{\circ}$ (energy in the range of

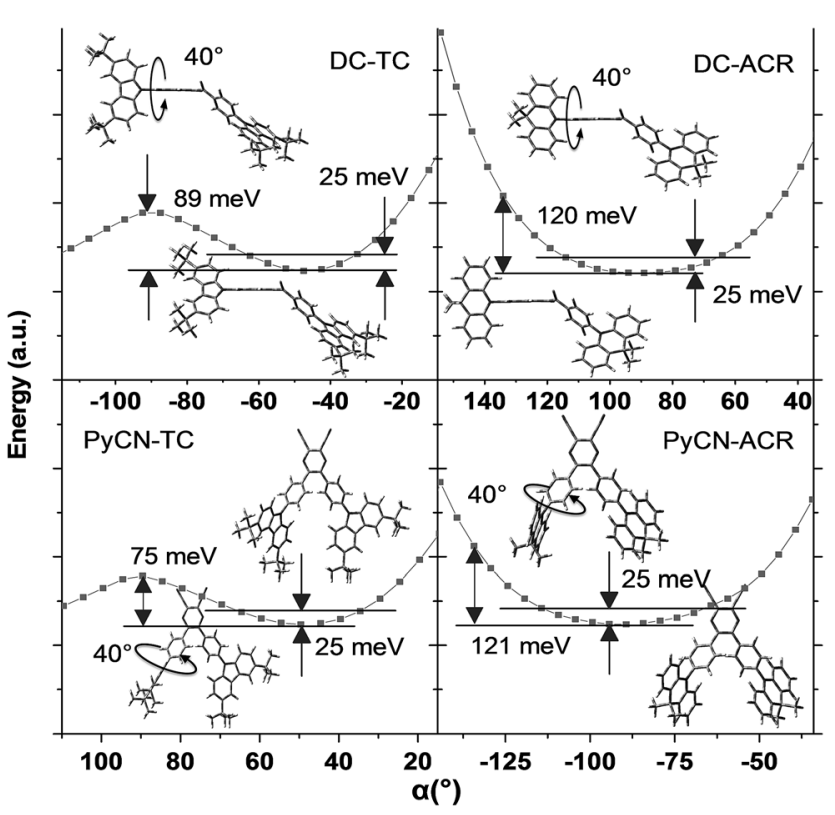

Fig. 5 Flexible potential surface scanning on the energy of the ground state geometry at different twisted angles $(\alpha)$ in toluene from BMK/6$31 \mathrm{G}$ *. The two solid lines label the thermally activated energy ( $25 \mathrm{meV}$ ) at $300 \mathrm{~K}$. The molecular insets represent the stationary point energyoptimized geometry (with $\alpha=40^{\circ}$ rotation) and the lowest energyoptimized geometry. 
$25 \mathrm{meV}$ ), the $f$ of ICT absorption asymptotically increases. In light of this, a moderate $k_{\mathrm{F}}$ and suppressed non-radiative process can be obtained simultaneously by the rigid but rotation-feasible ACR-Ph arms (Table 2).

For the photoluminescence (PL) spectra (Fig. 6 and S12 $\dagger$ ), when increasing the polarity of the solvent, a positive solvatochromism effect was observed. ${ }^{48}$ DC-TC and DC-ACR were hardly emissive while ring-locked PyCN-TC and PyCN-ACR exhibited higher PLQY in solution. After $\mathrm{N}_{2}$ treatment, the PLQYs of the four compounds in solution increase compared with those in air equilibrium solutions, implying that the dissolved oxygen quenches the triplet state excitons. ${ }^{49}$ As revealed in TD-DFT (Fig. 3), the less rigid DC-TC and DC-ACR undergo obvious conformation deformation, leading to a quick internal conversion (IC) process in $S_{1}$. When DC was chemically modified into PyCN, the increased rigidity significantly suppresses the non-radiative decay process and a higher PLQY can be obtained in liquid media. To verify such effects on restricted molecules, the PL of the four compounds in different water : THF mixtures ratio was measured (Fig. S13 $\dagger$ ). Higher water fractions increase the extent of aggregation, enabling suppression of non-radiative channels. ${ }^{50}$ Evidently, the emission of all the compounds was enhanced with increased aggregation when a high fraction of water was added.

In addition, the character of the triplet emission was analyzed via phosphorescence spectra at $77 \mathrm{~K}$. In good agreement with the TD-DFT analyses, the ${ }^{3} \mathrm{LE}$ triplet state of PyCN-TC was experimentally confirmed. The phosphorescence spectra of PyCN-TC suggested a specific vibrational structure. For the other three, the TD-DFT-simulated lowest ${ }^{3} \mathrm{CT}$ triplet states are verified by single ICT phosphorescence peaks. (Fig. S9†) $\Delta E_{\mathrm{ST}}$ of all the compounds can be calculated and the order is DC-ACR $(0.01 \mathrm{eV})>$ PyCN-ACR $(0.08 \mathrm{eV})>$ DC-TC $(0.13 \mathrm{eV})>$ PyCN-TC $(0.43 \mathrm{eV})$, in good agreement with the quantum simulation results.

To factually assess performance of the emitting materials in devices, the solid-state photo-physical properties of all the materials were investigated by doping them into $4,4^{\prime}$-bis(carbazol-9-yl)biphenyl (CBP). The doping concentration was controlled at a low level to avoid aggregation-induced quenching effects. The emission in the doped films blue-shifted by around $10-60 \mathrm{~nm}$ in contrast to that in solution, indicating that molecular reorganization and relaxation are hindered by the
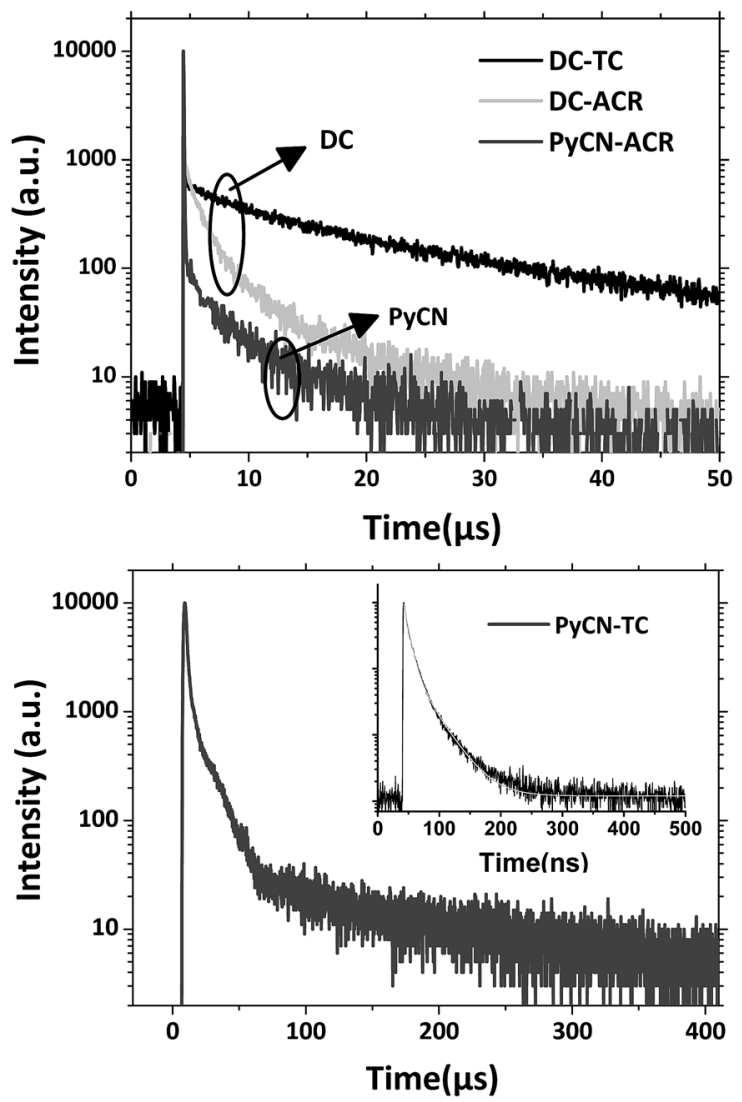

Fig. 6 Transient PL decay spectra of the compounds doped into CBP films (7 wt\%) measured with a fluorescence lifetime spectrometer (C11367-03, Hamamatsu Photonics) at room temperature in a $\mathrm{N}_{2}$ atmosphere.

surrounding solid matrix with a reduced Stokes bathochromic shift (Fig. S14†). ${ }^{15}$ To verify the TADF nature of all the materials, temperature-dependent transient PL spectra were measured from 77 to $350 \mathrm{~K}$ (Fig. 7 and S15-S17†). The delayed fluorescence ratio of all the materials showed enhancement when the temperature was increased, then some of the fluorescence ratios were followed by a slight decrease as the temperature was further increased to $350 \mathrm{~K}$. For PyCN-ACR, for example, the monotonically enhanced ratio of the delayed fluorescence from $9.7 \%$ to $60.5 \%$ firmly depicts the enhanced contribution of RISC

Table 2 Thermal, electrochemical and photo-physical properties of the investigated molecules

\begin{tabular}{|c|c|c|c|c|c|c|}
\hline Compound & $T_{\mathrm{d}}^{a} / T_{\mathrm{g}}{ }^{b}\left({ }^{\circ} \mathrm{C}\right)$ & $\mathrm{IP} / \mathrm{EA}^{c} / \mathrm{EA}^{d}(\mathrm{eV})$ & $E_{\mathrm{g}}{ }^{e} / E_{\mathrm{g}}^{f}(\mathrm{eV})$ & $\lambda_{\mathrm{abs}}(\mathrm{nm}) \mathrm{sol}^{g}$ & $\lambda_{\mathrm{PL}}(\mathrm{nm}) \mathrm{sol}^{g} / \mathrm{film}^{h}$ & PLQY (\%) sol ${ }^{g} /$ film $^{h}$ \\
\hline DC-TC & $413 / 78$ & $-5.56 /$ N.A./ -2.9 & N.A./2.58 & 389 & $553 / 518$ & $0.3(0.6) / 14.7(19.0)$ \\
\hline DC-ACR & $387 / 98$ & $-5.35 /$ N.A./ -2.84 & N.A./2.51 & 411 & $584 / 532$ & $0.8(2.0) / 11.5(13.0)$ \\
\hline PyCN-TC & 403/N.A. & $-5.56 /-3.32 /-3.26$ & $2.24 / 2.30$ & 429 & $532 / 534$ & $53.8(62.9) / 28.6(38.0)$ \\
\hline PyCN-ACR & 450/N.A. & $-5.33 /-3.18 /-3.17$ & $2.15 / 2.16$ & 438 & $618 / 560$ & $8.6(16.4) / 39.8(43.0)$ \\
\hline
\end{tabular}

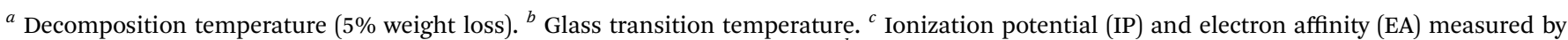

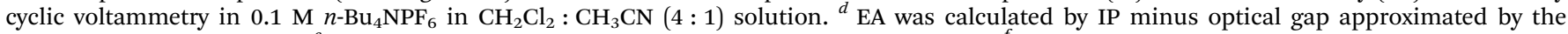

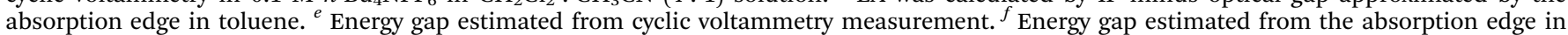

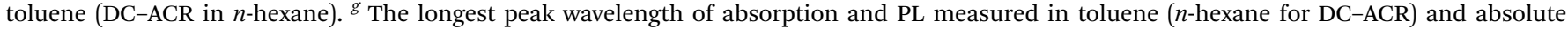

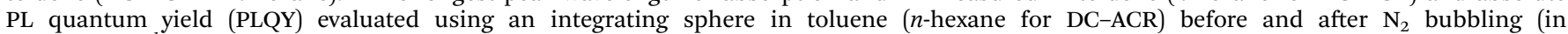
parenthesis). ${ }^{h}$ PLQY of $7 \mathrm{wt} \%$ investigated molecules doped into CBP in air and $\mathrm{N}_{2}$ atmosphere (in parenthesis), respectively. 


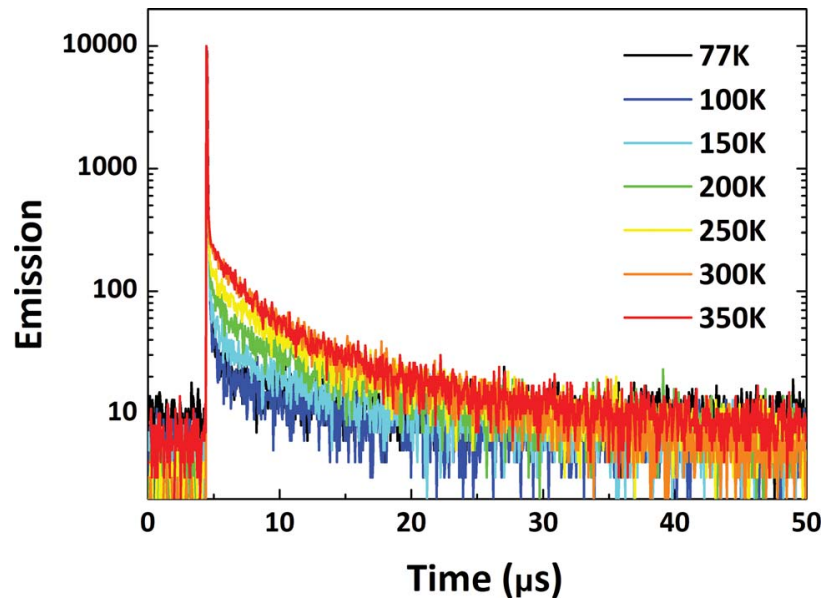

Fig. 7 Transient PL decay curves for 1 wt\% PyCN-ACR doped into CBP measured from 77 to $350 \mathrm{~K}$ in a $\mathrm{N}_{2}$ atmosphere.

from 77 to $300 \mathrm{~K}$. Conversely, further increasing the temperature from 300 to $350 \mathrm{~K}$ reduced the delayed fluorescence ratio from 60.5 to $56.2 \%$. Other than the suppressed IC process at low temperature, it competes significantly with RISC at high temperature and results in a reduced delay fluorescence ratio, which is one of the typical TADF characters. ${ }^{51}$ An analogous phenomenon was also observed for DC-TC, DC-ACR and PyCNTC, putting all of them into the category of TADF materials (Scheme 5).

Based on the transient PL decay curve at $300 \mathrm{~K}$ in a $\mathrm{N}_{2}$ atmosphere (Fig. 8), the prompt $\left(\Phi_{\mathrm{F}}\right)$ and delayed $\left(\Phi_{\mathrm{TADF}}\right)$ components of the PLQY as well as the prompt decay lifetime $\left(\tau_{\mathrm{p}}\right)$ and delayed decay lifetime $\left(\tau_{\mathrm{TADF}}\right)$ were separated for successive calculation. $k_{\mathrm{F}}, k_{\mathrm{ISC}}, k_{\mathrm{RISC}}$ and $k_{\mathrm{IC}}$ were estimated using the following formulas. ${ }^{8,15,52,53}$

$$
k_{\mathrm{F}}=\Phi_{\mathrm{F}} / \tau_{\mathrm{p}}
$$

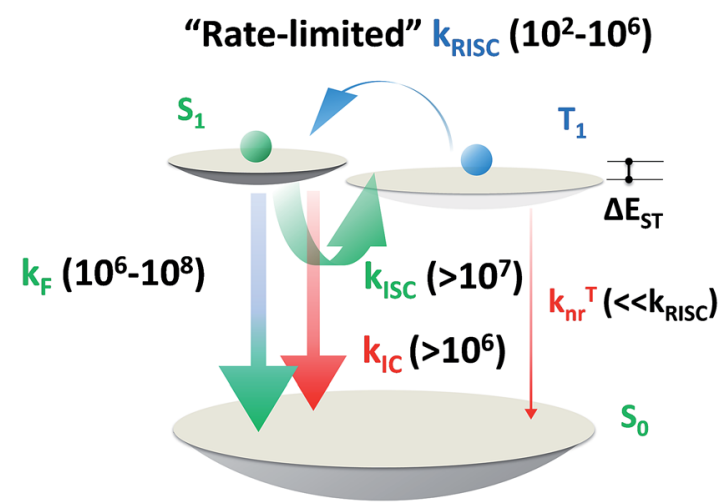

Scheme 5 Order of magnitude of rate constants involved in exciton dynamic processes for TADF emitters. As shown, $k_{\text {RIsC }}$ becomes the rate-limited step during these processes for tuning the lifetime of the excited state $\left(\tau_{\mathrm{TADF}}\right) . k_{\mathrm{F}}, k_{\mathrm{ISC}}, k_{\mathrm{IC}}$ and $k_{\mathrm{RISC}}$ are on the orders of $10^{6}$ to $10^{8},>10^{7},>10^{6}$ and $10^{2}$ to $10^{6}$ respectively, as summarized by previous reports. $^{7-9,13,15,51-54,56,58,61,64}$
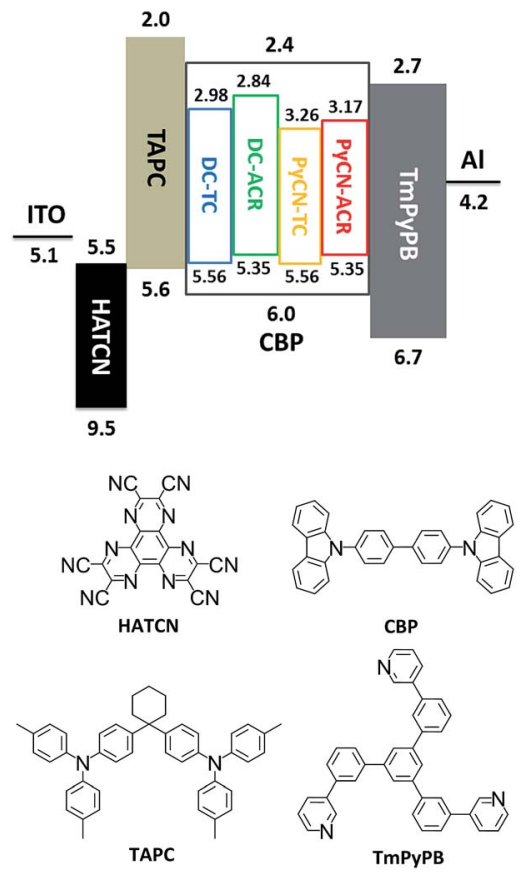

Fig. 8 Energy level diagram and molecular structures of the materials for fabricating OLED devices. The HOMO and LUMO levels of the investigated molecules were estimated by cyclic voltammetry.

$$
\begin{gathered}
\Phi_{\mathrm{ISC}}=1-\Phi_{\mathrm{F}} \\
\Phi_{\mathrm{F}}=k_{\mathrm{F}} /\left(k_{\mathrm{F}}+k_{\mathrm{ISC}}\right) \\
k_{\mathrm{RISC}}=\left(k_{\mathrm{p}} k_{\mathrm{d}} \Phi_{\mathrm{TADF}}\right) /\left(k_{\mathrm{ISC}} \Phi_{\mathrm{F}}\right) \\
\Phi=k_{\mathrm{F}} /\left(k_{\mathrm{F}}+k_{\mathrm{IC}}\right)
\end{gathered}
$$

As expected, the triplet yields ( $\left.\Phi_{\text {ISC }}\right)$ of DC-TC (93\%) and DCACR (97\%) are superior to those of PyCN-TC (67\%) and PyCNACR (80\%). Optically generated $S_{1}$ excitons are inclined to ISC into triplet states for DC-TC and DC-ACR because the $\mathrm{n}-\pi^{*}$ transition of carbonyl derivatives favours an ISC process. ${ }^{17}$ In contrast, when DC was chemically modified into PyCN, $\Phi_{\text {ISC }}$ and $k_{\text {ISC }}$ were reduced and an enhanced $k_{\mathrm{F}}$ was observed (PyCN-TC contrast to DC-TC, and PyCN-ACR contrast to DC-ACR, respectively). Owing to the similar $\alpha$ for DC-TC and PyCN-TC or DC-ACR and PyCN-ACR, the increased $\pi-\pi^{*}$ character induces a larger $k_{\mathrm{F}}$ for the latter pair. Additionally, when comparing the rigidity of DC-TC to PyCN-TC or DC-ACR to PyCN-ACR, lower $k_{\mathrm{IC}}$ values were observed for the latter owing to the more rigid PyCN core. Though PyCN-ACR shows a redder emission, the compact molecular structure results in the lowest $k_{\mathrm{IC}}$ among all the emitters. The $k_{\mathrm{RISC}}$ of DC-ACR and PyCN-ACR are an order of magnitude higher than that of DC-TC and PyCN-TC, indicating a more efficient RISC from $T_{1}$ to $S_{1}$ for the former pair. Interestingly, the delayed lifetimes of carbonyl-type DC-TC and DC-ACR differ greatly with analogous $\Phi_{\text {ISC }}$ ratios (over 90\%). In this case, the shortening $\tau_{\text {TADF }}$ of DC-ACR should be ascribed to a more efficient RISC process, and an even larger $k_{\mathrm{F}}$ for DC-TC 
$\left(0.56 \times 10^{7}\right)$ than DC-ACR $\left(0.13 \times 10^{7}\right)$ was observed. For $n-\pi^{*}$ favored DC-ACR and $\pi-\pi *$-favored PyCN-ACR with different $k_{\mathrm{ISC}}$ and $k_{\mathrm{F}}$ values, a shorter $\tau_{\mathrm{TADF}}$ was still observed for the former, with smaller $\Delta E_{\mathrm{ST}}$, reduced $k_{\mathrm{ISC}}$ and enhanced $k_{\mathrm{F}}$ contributing to the shortening of $\tau_{\mathrm{TADF}}$.

Based on a parallel comparison, as $k_{\mathrm{F}}, k_{\mathrm{ISC}}, k_{\mathrm{IC}}$ and $k_{\mathrm{RISC}}$ all contribute to $\tau_{\mathrm{TADF}}$ tuning, a large $k_{\mathrm{RISC}}$ plays the leading part. This can be interpreted by the "rate-limited effect" of the RISC process. For TADF emitters, the slow dissipation of triplet excitons and an inefficient RISC process can result in a rather long excited state lifetime. As $k_{\mathrm{F}}, k_{\mathrm{ISC}}$ and $k_{\mathrm{IC}}$ are larger than $10^{6}$ while $k_{\mathrm{RISC}}$ is from $10^{2}$ to $10^{6}, 7^{-9,54,55}$ the much smaller $k_{\mathrm{RISC}}$ becomes the rate-limited step in exciton dynamic processes. As the triplet state can be stable and its non-radiative process is much slower than RISC, the enhanced $k_{\text {RISC }}$ becomes critical because the up-converted triplet excitons can be deactivated quicker radiatively or non-radiatively in the $\mathrm{S}_{1}$ state. According to the analyses, a much shorter $\tau_{\text {TADF }}$ can be expected for DCACR even with a large $k_{\text {ISC }}$ and a small $k_{\mathrm{F}}$. For PyCN-TC, although the highest $k_{\mathrm{F}}$ and small $k_{\mathrm{ISC}}$ values are calculated, $\tau_{\text {TADF }}$ of PyCN-TC is much longer than the other three emitters considering it has the smallest $k_{\mathrm{RISC}}$. Based on the molecular design, we are able to figure that increasing $k_{\mathrm{RISC}}$ is the key to shortening $\tau_{\text {TADF }}$ (Table 3 ).

\section{OLED characterization}

After detailed investigation on the emitting properties and energy level relationships of the four TADF molecules, differences in the electroluminescence performance is investigated for the four materials. To reduce variants, the four TADF materials were employed in the same device architecture. Given that the carrier transport abilities of the investigated guest emitters have effects on the carrier balance and could influence the roll-off character of the devices, the doping concentration of the four TADF emitters were controlled at a low level ( $1 \mathrm{wt} \%$ for DC-ACR and PyCN-ACR, $5 \mathrm{wt} \%$ for DC-TC and PyCN-TC) but assuring complete energy transfer from host to guest. Consequently, the device efficiency roll-off can approximately be linked to the molecular characteristics. The fabricated device architecture is indium tin oxide (ITO)/HATCN (5 nm)/TAPC (20 $\mathrm{nm}) / x$ wt\% emitter in CBP $(35 \mathrm{~nm}) / \mathrm{TmPyPB}(55 \mathrm{~nm}) / \mathrm{LiF}(1 \mathrm{~nm}) /$ $\mathrm{Al}(100 \mathrm{~nm})$, in which the dipyrazino[2,3-f:20,30- $h]$ quinoxaline2,3,6,7,10,11-hexacarbonitrile (HATCN), (1,1-bis $(4-(N, N$-di $(p$ tolyl)-amino)-phenyl)cyclohexane) (TAPC), 1,3,5-tri( $m$-pyrid-3-ylphenyl)benzene (TmPyPB) and LiF layers play the roles of hole injection, hole transport and electron blocking, electron transport and hole blocking and electron injection layers, respectively. ${ }^{56}$ An energy level diagram is displayed in accordance with the cyclic voltammetry (CV) results (Fig. 8 and S23†). ${ }^{4}$ Unlike the inefficient energy transfer from host to guest under optical excitation (Fig. S14 $\dagger$ ), suppressed CBP host emission around $400 \mathrm{~nm}$ was noted in the EL spectra and additional energy capture channels should be considered. As the HOMO and LUMO of all guest molecules are shallower and deeper than that of CBP, relatively equilibrium hole and electron capture abilities can be obtained. ${ }^{57,58}$ Carriers can be trapped directly into the guest instead of solely transferring the energy via long-range Förster or short-range Dexter transfer, leading to complete energy transfer from host to guest in contrast to those under optical excitation (Fig. S14†). ${ }^{\mathbf{9 9 , 6 0}}$

Current density-voltage-luminance $(J-V-L)$, luminance efficiency-luminance-power efficiency (LE- $L-\mathrm{PE}$ ), EQE-luminance (EQE-L) characteristics and EL spectra are listed in Fig. 9. From the EL spectra, emission peaks from greenish-yellow to orange locating at $538 \mathrm{~nm}$ for DC-TC, $546 \mathrm{~nm}$ for DC-ACR and PyCNTC, and $572 \mathrm{~nm}$ for PyCN-ACR are observed. The turn-on voltages of the four materials are about the same at 3.4 to $3.5 \mathrm{~V}$, detected at the luminance of $1 \mathrm{~cd} \mathrm{~m}^{-2}$. The maximum EQEs of the four devices are 6.2\% (DC-TC), 6.3\% (DC-ACR), 8.1\% (PyCN-TC) and 15.6\% (PyCN-ACR). Applications of benzil derivatives in OLEDs have been deemed to be impractical due to their poor PLQY. However, the achieved EQEs exceeding the 5\% theoretical limitation of traditional fluorophores suggests the efficient utilization of $75 \%$ triplet excitons generated under electric excitation.

Different degrees of efficiency roll-off at high current density were observed, eliminating the possibility of a TTA mechanism leading to the high EQE. Among the four emitters, PyCN-TC exhibits the largest $\Delta E_{\mathrm{ST}}$ and ${ }^{3} \mathrm{LE}$ triplet state, eventuating in $\tau_{\text {TADF }}$ exceeding $200 \mu \mathrm{s}$. The hardly up-converted $\mathrm{T}_{1}$ exciton contributes less to delayed fluorescence and leads to a high triplet density population even with a high $k_{\mathrm{F}}$ and a small $k_{\mathrm{ISC}}$. The most significant efficiency roll-off was observed with only $23 \%$ and $11 \%$ of $\mathrm{EQE}_{\max }$ remaining at luminances of 100 and $1000 \mathrm{~cd} \mathrm{~m}^{-2}$ respectively.

Towards DC-TC, DC-ACR and PyCN-ACR with the lowest ${ }^{3} \mathrm{CT}$ triplet states, efficiency roll-off can also be linked to $\tau_{\mathrm{TADF}}$. As mentioned, the enhanced $k_{\mathrm{RISC}}$ effectively shortens $\tau_{\mathrm{TADF}}$, endowing reduced triplet exciton density for suppressed STA and TTA. For DC-TC, owing to the larger $\Delta E_{\mathrm{ST}}$ than DC-ACR and PyCN-ACR, a much longer $\tau_{\text {TADF }}$ increased the ISC-RISC

Table 3 Photo-physical data of the investigated molecules doped into CBP films (7 wt\%) at room temperature

\begin{tabular}{|c|c|c|c|c|c|c|c|c|c|c|c|c|}
\hline Compound & $\begin{array}{l}\lambda_{\mathrm{em}} \\
(\mathrm{nm})\end{array}$ & $\Phi$ & $\Phi_{\mathrm{F}}$ & $\Phi_{\mathrm{TADF}}$ & $\Phi_{\mathrm{ISC}}$ & $\begin{array}{l}\tau_{\mathrm{p}} \\
(\mathrm{ns})\end{array}$ & $\begin{array}{l}\tau_{\mathrm{TADF}}{ }^{a} \\
(\mu \mathrm{S})\end{array}$ & $\begin{array}{l}k_{\mathrm{F}} \\
\left(\times 10^{7} \mathrm{~s}^{-1}\right)\end{array}$ & $\begin{array}{l}k_{\mathrm{IC}} \\
\left(\times 10^{7} \mathrm{~s}^{-1}\right)\end{array}$ & $\begin{array}{l}k_{\mathrm{ISC}} \\
\left(\times 10^{7} \mathrm{~s}^{-1}\right)\end{array}$ & $\begin{array}{l}k_{\mathrm{RISC}} \\
\left(\times 10^{5} \mathrm{~s}^{-1}\right)\end{array}$ & $\begin{array}{l}\Delta E_{\mathrm{ST}} \\
(\mathrm{eV})\end{array}$ \\
\hline DC-TC & 518 & 0.19 & 0.07 & 0.12 & 0.93 & 12.6 & 24.9 & 0.56 & 2.4 & 7.4 & 0.74 & 0.13 \\
\hline DC-ACR & 532 & 0.13 & 0.03 & 0.10 & 0.97 & 22.8 & 3.0 & 0.13 & 0.9 & 4.3 & 11.4 & 0.01 \\
\hline PyCN-TC & 534 & 0.38 & 0.33 & 0.05 & 0.67 & 25.2 & 210.5 & 1.31 & 2.1 & 2.60 & 0.011 & 0.43 \\
\hline PyCN-ACR & 575 & 0.43 & 0.20 & 0.23 & 0.80 & 34.3 & 4.6 & 0.58 & 0.7 & 2.32 & 3.13 & 0.08 \\
\hline
\end{tabular}

${ }^{a}$ Average lifetime calculated by $\tau_{\mathrm{av}}=\Sigma A_{\mathrm{i}} \tau_{\mathrm{i}}{ }^{2} / \Sigma A_{\mathrm{i}} \tau_{\mathrm{i}}$, where $A_{\mathrm{i}}$ is the pre-exponential for lifetime $\tau_{\mathrm{i}}$. 

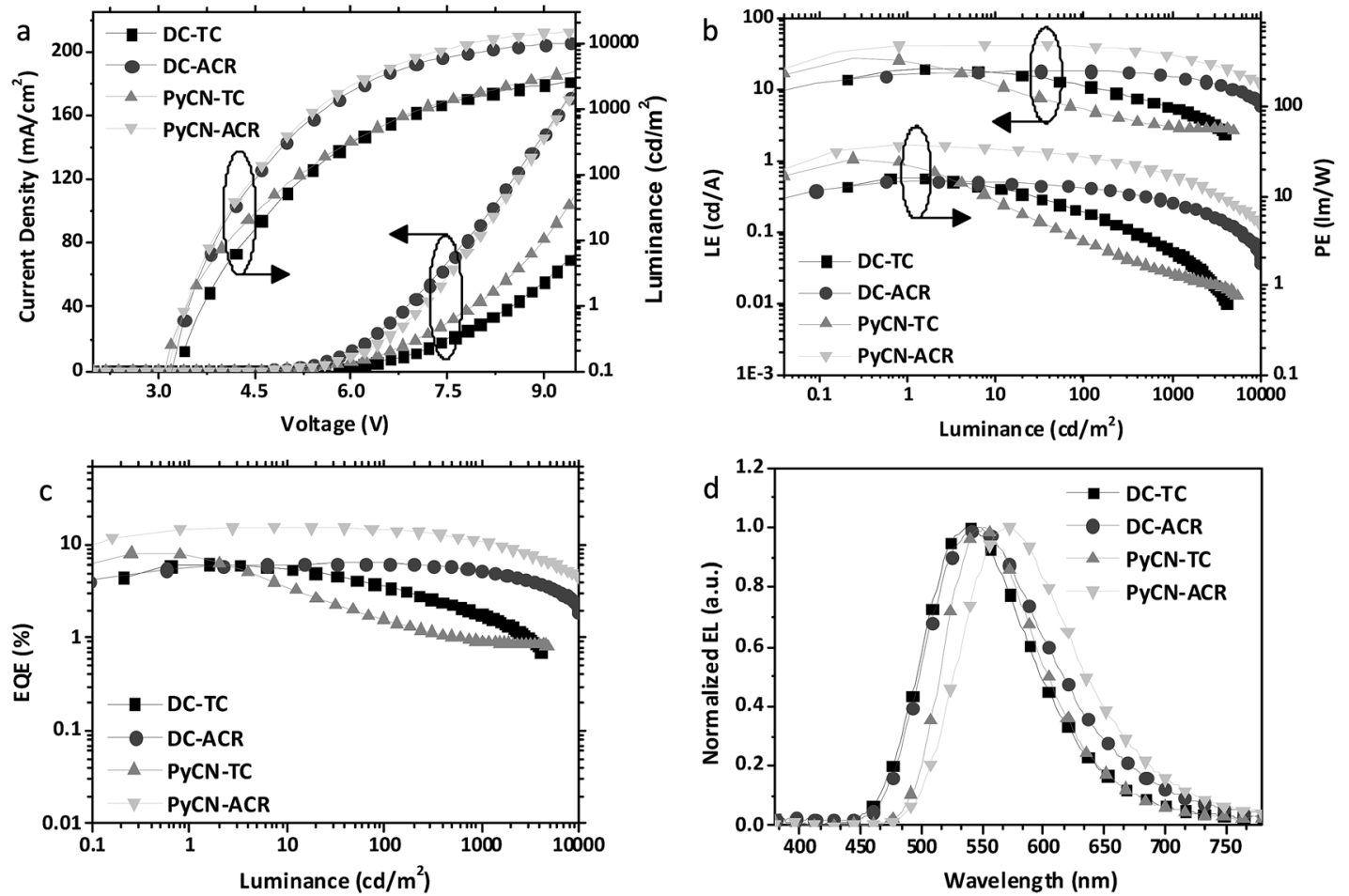

Fig. 9 (a) Current density-voltage-luminance $(J-V-L)$, (b) luminance efficiency-luminance-power efficiency (LE-L-PE), (c) EQE-luminance characteristics, and (d) EL spectra at $10 \mathrm{~mA} \mathrm{~cm}^{-2}$ of the devices based on the investigated molecules.

cycles between radiative $S_{1}$ and non-radiative $T_{1}$, resulting in a remarkable efficiency roll-off with only $37.1 \%$ of the $\mathrm{EQE}_{\max }$ remaining at a luminance of $1000 \mathrm{~cd} \mathrm{~m}^{-2}$. In contrast, a wellcontrolled roll-off was achieved for DC-ACR with the shortest $\tau_{\text {TADF }}$ among four emitters, exhibiting $100 \%$ and $81 \%$ of the $\mathrm{EQE}_{\text {max }}$ at luminances of 100 and $1000 \mathrm{~cd} \mathrm{~m}^{-2}$ respectively. In spite of the close $\Phi_{\text {ISC }}$ ratio for DC-TC and DC-ACR and the even larger $k_{\mathrm{F}}$ for the former, an enhanced RISC process plays the dominant role in reducing the efficiency roll-off for TADFOLEDs. For PyCN-ACR with a larger $k_{\text {RISC }}$ than DC-TC, a lower device efficiency roll-off is also observed at practical brightness. Note that different doping concentrations of the guest could influence the efficiency roll-off of the device; devices with the same guest doping concentration $(5 \mathrm{wt} \%)$ were also fabricated for comparison (Fig. S20 ${ }^{\dagger}$ ). For TADF-OLEDs using DC-ACR and PyCN-ACR as the emitters, the tendency is consistent with our analyses. Emitters with a large $k_{\text {RISC }}$ tended to exhibit low efficiency roll-off character at practical brightness (Fig. 10).

Further information on the mechanism of large $k_{\mathrm{RISC}}$ for reduced efficiency roll-off can be found by fitting the EQE- $J$ curve with the EQE roll-off model of TADF-OLEDs (Fig. S22 $\dagger$ ). A high singlet exciton concentration from efficient RISC, STA and TTA processes can be the dominant source for exciton annihilation. ${ }^{\mathbf{1 3 6 1 , 6 2}}$ For a steady-state current, the model EQEs are fitted and afford the rate constants of STA $\left(k_{\mathrm{ST}}\right)$ and TTA $\left(k_{\mathrm{TT}}\right)$. Very small HONTO and LUNTO overlap for PyCN-ACR and DC-ACR renders a small $f$ and the rate constant of Förster energy transfer-type STA could be low. Though the calculated $k_{\mathrm{TT}}$ are two magnitudes smaller than $k_{\mathrm{ST}}$, the triplet density can be over three orders higher than the singlet density and TTA could aggravate the efficiency roll-off of the device. ${ }^{13,63}$ In good agreement of our analyses, the $k_{\mathrm{ST}}$ and $k_{\mathrm{TT}}$ of DC-ACR and PyCN-ACR are much smaller than those of the other two emitters with longer $\tau_{\text {TADF }}$, indicating more suppressed STA and TTA for OLEDs using emitters with a large $k_{\text {RISC }}$ and a short $\tau_{\text {TADF }}$.

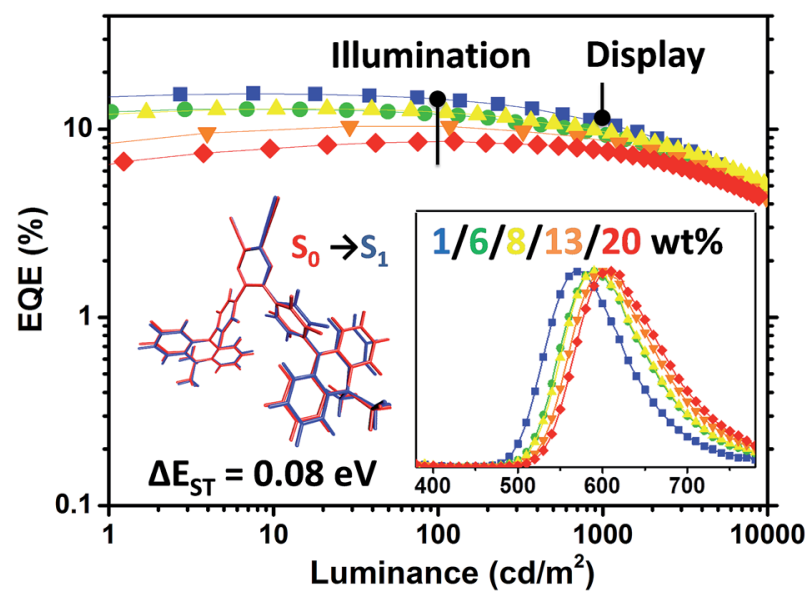

Fig. 10 EQE-luminance curves of the OLED devices based on PyCNACR at various doping concentrations in a structure of ITO/HATCN (5 nm)/TAPC (20 nm)/CBP: $x$ wt\% PyCN-ACR (35 nm)/TmPyPB (55 nm)/ LiF $(1 \mathrm{~nm}) / \mathrm{Al}$. Inset: (left) small geometry deformation in the excited state and $\Delta E_{\mathrm{ST}}$ for PyCN-ACR are responsible for highly efficient and low roll-off orange-red TADF-OLEDs; (right) EL spectra of the corresponding devices at $10 \mathrm{~mA} \mathrm{~cm}{ }^{-2}$ with different doping concentrations of PyCN-ACR in CBP. 
Notably, the $1 \mathrm{wt} \%$ PyCN-ACR in CBP device exhibits orange $\mathrm{EL}$ at $572 \mathrm{~nm}$. The slightly bathochromic shift $(12 \mathrm{~nm})$ with respect to the PL in the doped film implies geometry relaxation in the solid matrix. When the doping concentration was adjusted from $1 \mathrm{wt} \%$ to $20 \mathrm{wt} \%$, a red-shift as large as $34 \mathrm{~nm}$ was observed. Under an electric field, TADF molecules with a large dipole moment tend to polarize in the host medium. Such a "solvatochromic" shift in the solid matrix can be observed when TADF emitters are doped into bipolar hosts or the doping concentration of the guest is increased. ${ }^{\mathbf{6 4 , 6 5}} \mathrm{A}$ low efficiency rolloff was noted for all the devices with different doping concentrations. The maximum efficiency roll-off turned out to be $38.8 \%$ at $1000 \mathrm{~cd} \mathrm{~m}^{-2}$ for $1 \mathrm{wt} \%$ PyCN-ACR doped into CBP. Especially for the devices with doping concentrations of $8 \mathrm{wt} \%$ and $13 \mathrm{wt} \%$, EQEs of $12.4 \%$ and $10.3 \%$ at a luminance of $100 \mathrm{~cd}$ $\mathrm{m}^{-2}$ as well as EQEs of $9.9 \%$ and $8.8 \%$ at a luminance of $1000 \mathrm{~cd}$ $\mathrm{m}^{-2}$ were achieved. The roll-off was less than $25 \%$ and $15 \%$ and emission peaks were located at 590 and $600 \mathrm{~nm}$, respectively. Even at a luminance of $10000 \mathrm{~cd} \mathrm{~m}^{-2}$, EQEs as high as 5.1\% and $4.4 \%$ were maintained. For $\mathrm{PyCN}-\mathrm{ACR}$, the rotationfeasible ACR-Ph arms, enhanced $\pi-\pi^{*}$ feature and compact molecular geometry endow a moderate $\Phi$ and restricted nonradiative channel for orange-red emitters with a narrow band gap. In combination with the high $k_{\mathrm{RISC}}$, a low efficiency roll-off and highly efficient orange-red device employing PyCN-ACR can be realized, achieving the best EQE of orange-red TADFOLEDs at application relevant brightness (100/1000/10 $000 \mathrm{~cd}$ $\mathrm{m}^{-2}$ ) without employing host management.

\section{Conclusions}

Via molecular design, the effects of $k_{\mathrm{F}}, k_{\mathrm{ISC}}, k_{\mathrm{IC}}$ and $k_{\mathrm{RISC}}$ on $\tau_{\text {TADF }}$ governing were investigated based on four representative TADF emitters. $\tau_{\text {TADF }}$ can be effectively shortened by enhanced RISC (small $\Delta E_{\mathrm{ST}}$ ) even with a small $k_{\mathrm{F}}$ and a large $k_{\mathrm{ISC}}$. As RISC is slower than other exciton dynamic processes and $T_{1}$ is relatively stable, it becomes the rate-limited step in shortening $\tau_{\text {TADF }}$ ("rate-limited effect" of the RISC process). When TADF materials with short $\tau_{\text {TADF }}$ are employed in OLEDs, more suppressed efficiency roll-off at practical relevant brightnesses (100/ $1000 / 10000 \mathrm{~cd} \mathrm{~m}^{-2}$ ) is observed. The shortened $\tau_{\text {TADF }}$ indicates a more efficient RISC process and eventuates in reduced triplet exciton density for suppressed STA and TTA.

Following the aforementioned guidelines, TADF materials should be carefully designed to govern $\tau_{\mathrm{TADF}}$. In light of the critical role of $k_{\mathrm{RISC}}$, factors concerning tuning $\Delta E_{\mathrm{ST}}$ can effectively alter $\tau_{\mathrm{TADF}}$. One of the approaches is tuning the $\mathrm{D}-\mathrm{A}$ strength of the emitters for enhanced ICT, which can render small frontier orbital overlap and reduce $\Delta E_{\mathrm{ST}}$. A more promising way is tuning the dihedral angles between electron donating and withdrawing moieties in TICT molecular systems. The enlarged dihedral angle induces separated HOMO/LUMO overlap while a flat D-A structure does the opposite. The former case reduces $\Delta E_{\mathrm{ST}}$ and effectively shortens $\tau_{\mathrm{TADF}}$, giving these materials potential for fabricating low efficiency roll-off OLED devices. The latter case can elongate $\tau_{\text {TADF }}$, making these materials promising for ultra-long lifetime delayed fluorescence applications such as bio-imaging, ${ }^{66}$ oxygen sensors ${ }^{67}$ or antifake, ${ }^{68}$ etc. Finally, tuning ${ }^{3} \mathrm{LE}$ and ${ }^{3} \mathrm{CT}$ coupling also helps to tune $\tau_{\text {TADF }}$. For TADF-OLEDs, materials with ${ }^{3} \mathrm{LE}$ can be detrimental for shortening $\tau_{\text {TADF }}$ because the additional RIC process makes RISC tougher. OLEDs using such emitters exhibit significant roll-off, and a low EQE is expected at high brightness. Chemical moieties for fabricating TADF emitters should not have low triplet states to make sure that the locally excited state lies above the CT channel.

\section{Acknowledgements}

The authors greatly appreciate the financial support from the Ministry of Science and Technology (2015CB655003 and 2014DFA52030), the National Natural Science Foundation of China (91233116 and 51573059), and Guangdong Provincial Department of Science and Technology (2014B090901048).

\section{Notes and references}

1 M. A. Baldo, D. O'brien, Y. You, A. Shoustikov, S. Sibley, M. Thompson and S. Forrest, Nature, 1998, 395, 151-154.

2 Y. Ma, H. Zhang, J. Shen and C. Che, Synth. Met., 1998, 94, 245-248.

3 Z. H. Kafafi, Organic electroluminescence, CRC Press, 2005.

4 M. A. Baldo, C. Adachi and S. R. Forrest, Phys. Rev. B: Condens. Matter Mater. Phys., 2000, 62, 10967-10976.

5 S. J. Su, T. Chiba, T. Takeda and J. Kido, Adv. Mater., 2008, 20, 2125-2130.

6 A. Endo, M. Ogasawara, A. Takahashi, D. Yokoyama, Y. Kato and C. Adachi, Adv. Mater., 2009, 21, 4802-4806.

7 S. Hirata, Y. Sakai, K. Masui, H. Tanaka, S. Y. Lee, H. Nomura, N. Nakamura, M. Yasumatsu, H. Nakanotani, Q. Zhang, K. Shizu, H. Miyazaki and C. Adachi, Nat. Mater., 2015, 14, 330-336.

8 H. Uoyama, K. Goushi, K. Shizu, H. Nomura and C. Adachi, Nature, 2012, 492, 234-238.

9 Q. Zhang, B. Li, S. Huang, H. Nomura, H. Tanaka and C. Adachi, Nat. Photonics, 2014, 8, 326-332.

10 M. Liu, Y. Seino, D. Chen, S. Inomata, S.-J. Su, H. Sasabe and J. Kido, Chem. Commun., 2015, 51, 16353-16356.

11 D. Chen, G. Xie, X. Cai, M. Liu, Y. Cao and S.-J. Su, Adv. Mater., 2016, 28, 239-244.

12 K.-C. Tang, K. L. Liu and I. C. Chen, Chem. Phys. Lett., 2004, 386, 437-441.

13 K. Masui, H. Nakanotani and C. Adachi, Org. Electron., 2013, 14, 2721-2726.

14 N. J. Turro, V. Ramamurthy and J. C. Scaiano, Modern molecular photochemistry of organic molecules, University Science Books, Sausalito, CA, 2010.

15 Q. Zhang, H. Kuwabara, W. J. Potscavage Jr, S. Huang, Y. Hatae, T. Shibata and C. Adachi, J. Am. Chem. Soc., 2014, 136, 18070-18081.

16 T. Itoh, Chem. Phys. Lett., 1988, 151, 166-168.

17 D. R. Kearns and W. A. Case, J. Am. Chem. Soc., 1966, 88, 5087-5097.

18 T. Förster, Discuss. Faraday Soc., 1959, 27, 7. 
19 Y. Tao, K. Yuan, T. Chen, P. Xu, H. Li, R. Chen, C. Zheng, L. Zhang and W. Huang, Adv. Mater., 2014, 26, 7931-7958.

20 A. Endo, K. Sato, K. Yoshimura, T. Kai, A. Kawada, H. Miyazaki and C. Adachi, Appl. Phys. Lett., 2011, 98, 083302.

21 M. W. Wolf, K. D. Legg, R. E. Brown, L. A. Singer and J. H. Parks, J. Am. Chem. Soc., 1975, 97, 4490-4497.

22 Y. P. Sun, D. F. Sears Jr and J. Saltiel, J. Am. Chem. Soc., 1989, 111, 706-711.

23 S. Y. Lee, T. Yasuda, Y. S. Yang, Q. Zhang and C. Adachi, Angew. Chem., Int. Ed., 2014, 53, 6402-6406.

24 B. Gao, Q. Zhou, Y. Geng, Y. Cheng, D. Ma, Z. Xie, L. Wang and F. Wang, Mater. Chem. Phys., 2006, 99, 247-252.

25 S. Chen, X. Xu, Y. Liu, G. Yu, X. Sun, W. Qiu, Y. Ma and D. Zhu, Adv. Funct. Mater., 2005, 15, 1541-1546.

26 M. A. Marques and E. Gross, Annu. Rev. Phys. Chem., 2004, 55, 427-455.

27 E. Runge and E. K. Gross, Phys. Rev. Lett., 1984, 52, 997.

28 M. Reiher, O. Salomon and B. A. Hess, Theor. Chem. Acc., 2001, 107, 48-55.

29 D. Becke, J. Chem. Phys., 1993, 98, 5648-5652.

30 R. Magyar and S. Tretiak, J. Chem. Theory Comput., 2007, 3, 976-987.

31 A. Dreuw and M. Head-Gordon, Chem. Rev., 2005, 105, 40094037.

32 A. D. Boese and J. M. L. Martin, J. Chem. Phys., 2004, 121, 3405-3416.

33 R. Improta, V. Barone, G. Scalmani and M. J. Frisch, J. Chem. Phys., 2006, 125, 054103.

34 P. H. Harbach and A. Dreuw, in Modeling of Molecular Properties, ed. P. Comba, Wiley-VCH, Weinheim, 2011, pp. 37-38.

35 D. Kim, V. Coropceanu and J.-L. Brédas, J. Am. Chem. Soc., 2011, 133, 17895-17900.

36 S. Salman, D. Kim, V. Coropceanu and J.-L. Brédas, Chem. Mater., 2011, 23, 5223-5230.

37 Z. R. Grabowski, K. Rotkiewicz, A. Siemiarczuk, D. Cowley and W. Baumann, Nouv. J. Chim., 1979, 3, 443-454.

38 M. Maus and W. Rettig, Chem. Phys., 1997, 218, 151-162.

39 M. Maus, W. Rettig, G. Jonusauskas, R. Lapouyade and C. Rullière, J. Phys. Chem. A, 1998, 102, 7393-7405.

40 M. Maus, W. Rettig, D. Bonafoux and R. Lapouyade, J. Phys. Chem. A, 1999, 103, 3388-3401.

41 D. Jacquemin, A. Planchat, C. Adamo and B. Mennucci, J. Chem. Theory Comput., 2012, 8, 2359-2372.

42 S. Huang, Q. Zhang, Y. Shiota, T. Nakagawa, K. Kuwabara, K. Yoshizawa and C. Adachi, J. Chem. Theory Comput., 2013, 9, 3872-3877.

43 Q. Zhang, J. Li, K. Shizu, S. Huang, S. Hirata, H. Miyazaki and C. Adachi, J. Am. Chem. Soc., 2012, 134, 14706-14709.

44 Z. R. Grabowski, K. Rotkiewicz and W. Rettig, Chem. Rev., 2003, 103, 3899-4032.
45 W. Li, D. Liu, F. Shen, D. Ma, Z. Wang, T. Feng, Y. Xu, B. Yang and Y. Ma, Adv. Funct. Mater., 2012, 22, 2797-2803.

46 L. Yao, Y. Pan, X. Tang, Q. Bai, F. Shen, F. Li, P. Lu, B. Yang and Y. Ma, J. Phys. Chem. C, 2015, 119, 17800-17808.

47 T. Lu and F. Chen, J. Comput. Chem., 2012, 33, 580-592.

48 P. Wang and S. Wu, J. Photochem. Photobiol., A, 1995, 86, 109113.

49 T. Serevicius, T. Nakagawa, M. C. Kuo, S. H. Cheng, K. T. Wong, C. H. Chang, R. C. Kwong, S. Xia and C. Adachi, Phys. Chem. Chem. Phys., 2013, 15, 15850-15855.

50 L. Yao, S. Zhang, R. Wang, W. Li, F. Shen, B. Yang and Y. Ma, Angew. Chem., Int. Ed., 2014, 53, 2119-2123.

51 J. Li, T. Nakagawa, J. MacDonald, Q. Zhang, H. Nomura, H. Miyazaki and C. Adachi, Adv. Mater., 2013, 25, 3319-3323.

52 K. Goushi, K. Yoshida, K. Sato and C. Adachi, Nat. Photonics, 2012, 6, 253-258.

53 H. Tanaka, K. Shizu, J. Lee and C. Adachi, J. Phys. Chem. C, 2015, 119, 2948-2955.

54 J. Li, Q. Zhang, H. Nomura, H. Miyazaki and C. Adachi, Appl. Phys. Lett., 2014, 105, 013301.

55 M. Numata, T. Yasuda and C. Adachi, Chem. Commun., 2015, 51, 9443-9446.

56 G. Xie, X. Li, D. Chen, Z. Wang, X. Cai, D. Chen, Y. Li, K. Liu, Y. Cao and S.-J. Su, Adv. Mater., 2016, 28, 181-187.

57 S. J. Su, E. Gonmori, H. Sasabe and J. Kido, Adv. Mater., 2008, 20, 4189.

58 D. Zhang, L. Duan, C. Li, Y. Li, H. Li, D. Zhang and Y. Qiu, Adv. Mater., 2014, 26, 5050-5055.

59 H. Nakanotani, T. Higuchi, T. Furukawa, K. Masui, K. Morimoto, M. Numata, H. Tanaka, Y. Sagara, T. Yasuda and C. Adachi, Nat. Commun., 2014, 5, 4016.

60 M. Baldo, M. Thompson and S. Forrest, Nature, 2000, 403, 750-753.

61 D. Kasemann, R. Brückner, H. Fröb and K. Leo, Phys. Rev. B: Condens. Matter Mater. Phys., 2011, 84, 115208.

62 Y. Zhang and S. R. Forrest, Phys. Rev. Lett., 2012, 108, 267404.

63 T. Furukawa, H. Nakanotani, M. Inoue and C. Adachi, Sci. Rep., 2015, 5, 8429.

64 G. Méhes, K. Goushi, W. J. Potscavage and C. Adachi, Org. Electron., 2014, 15, 2027-2037.

65 C. F. Madigan and V. Bulović, Phys. Rev. Lett., 2003, 91, 247403.

66 X. Xiong, F. Song, J. Wang, Y. Zhang, Y. Xue, L. Sun, N. Jiang, P. Gao, L. Tian and X. Peng, J. Am. Chem. Soc., 2014, 136, 9590-9597.

67 S. Nagl, C. Baleizao, S. M. Borisov, M. Schaferling, M. N. Berberan-Santos and O. S. Wolfbeis, Angew. Chem., Int. Ed., 2007, 46, 2317-2319.

68 S. Hirata, K. Totani, J. Zhang, T. Yamashita, H. Kaji, S. R. Marder, T. Watanabe and C. Adachi, Adv. Funct. Mater., 2013, 23, 3386-3397. 